PAPER

\title{
Factors predicting improvement in motor disability in writer's cramp treated with botulinum toxin
}

\author{
R Djebbari, S T du Montcel, S Sangla, J S Vidal, G Gallouedec, M Vidailhet
}

J Neurol Neurosurg Psychiatry 2004;75:1688-1691. doi: 10.1136/jnnp.2003.032227

See end of article for authors' affiliations

......................

Correspondence to: Dr M Vidailhet, Service de Neurologie, Hôpital Saint Antoine, 184 rue du Faubourg Saint Antoine, 75012 Paris, France; marie.vidailhet@ sat.ap-hop-paris.fr

Received

11 November 2003

Revised 26 January 2004

Accepted

26 February 2004
Objective: To identify factors predicting improvement in motor disability in writer's cramp treated with botulinum toxin (BTX).

Methods: 47 patients with writer's cramp were treated with BTX and were evaluated by the same neurologists at initial referral, after each BTX injection, and when the effect of BTX was maximal at the time of the study. Patients and examiners simultaneously and independently rated the efficacy of BTX injections. Self assessment was a global clinical impression of the impact of treatment on writing quality, writing speed, writing errors, and legibility of handwriting; for objective assessment, the examiners used the Burke-Fahn-Marsden (BFM) scale.

Results: On the BFM scale, there was a significant improvement $(p<0.0001)$ in both severity and disability scores. Patients with a pronation/flexion pattern of dystonia showed the best and the most sustained improvement. Primary writing tremor was little improved. There was a correlation between the self assessment score and the Burke-Fahn-Marsden score. Benefit was maintained over time

Conclusions: These results have implications for the identification of patients most likely to benefit from BTX injections.
W riter's cramp, a focal hand dystonia, is highly disruptive because it interferes with professional and personal life. ${ }^{1}$ To date, although several therapeutic studies have been conducted with botulinum toxin (BTX), ${ }^{2-11}$ data are still needed to determine the best therapeutic strategies. The main difficulty is to distinguish in advance the patients who will benefit from BTX. Our aim in this study was to identify the predictive factors for improvement in motor disability in a group of patients with writer's cramp treated with BTX injections.

\section{METHODS}

Forty seven patients with writer's cramp were treated with BTX between January 1998 and December 2002 at the movement disorders clinic of Saint-Antoine Hospital, Paris. All the patients were evaluated by the same neurologists (RD, SS, MV) when they were initially referred ("first visit"), after each BTX injection (routine follow up), and at the time of the study, when all the patients were consecutively evaluated between January and December 2002. At that time, evaluation was obtained at the point of maximal effect of the injection ("last visit"). For the routine follow up visits, the patients were evaluated as they came for their usual evaluation visit, before the BTX injection session. Overall, the patients were examined every four to five months.

Inclusion criteria for BTX injections were:

- the presence of disabling writer's cramp (including primary writing tremor);

- the identification of a predominant abnormal posture or abnormal movement responsible for the difficulty in writing.

Writer's cramp was defined as a task specific dystonia triggered by writing, with no impairment of any other motor task. Tremor or myoclonus could be superimposed on the dystonic spasms or could be the main feature of the writer's cramp. Primary writing tremor was defined as task specific dystonic tremor-a tremor that appeared only during writing (producing a tremulous irregular handwriting) without any postural tremor or action tremor under any other conditions. In those patients, as most of the arm and hand muscles were involved, the aim of the clinical examination was to detect a predominant agonist-antagonist flexion-extension or pronation-supination pattern, in order to inject the corresponding muscles.

In patients with dystonic writer's cramp, the abnormal movements and posture were observed as the patient held the pen with the dominant hand and then wrote repeatedly (at least five times) the same sentence ("je respire le doux parfum des fleurs"). Abnormal dystonic patterns were described as flexion of the wrist, flexion-pronation of the forearm, supination of the forearm, extension of the wrist, flexion of the fingers, forced flexion of the thumb and index finger, extension of the thumb and index finger, extension of the fingers, or shoulder elevation. The presence of mirror movements in the relaxed dominant hand while writing with the non-dominant hand was explored systematically. It was considered to be a valuable clue when the mirror movements partially or completely reproduced a dystonic posture in the dominant hand (similar to that observed when writing with this hand). A specific motor pattern (for example, flexionpronation of the forearm) was the result of combined activation of various muscles (such as the flexor carpi radialis or pronator teres), even though other muscles could play an accessory role (overflow to the flexor carpi ulnaris or the flexor digitorum profundus). Thus the identification of the muscles most engaged in this pattern was obtained by clinical evaluation and led to the selection of the muscles that required BTX injections. The doses corresponding to each muscle were standardised and were not modified thereafter (table 1). For all muscles, electromyographic guidance for the BTX injections was used to increase the accuracy of muscle localisation and the selectivity of the BTX treatment. ${ }^{12}$

Abbreviations: BFM, Burke-Fahn-Marsden scale; BTX, botulinum toxin 
Table 1 The muscles most often injected with botulinum toxin

\begin{tabular}{|c|c|c|c|}
\hline & Muscles & $\begin{array}{l}\text { Per cent of total } \\
\text { number of injections }\end{array}$ & $\begin{array}{l}\text { Standardised dose of } \\
\text { botulinum toxin }\end{array}$ \\
\hline Flexion of the wrist & $\begin{array}{l}\text { Flexor carpi ulnaris } \\
\text { Flexor carpi radialis }\end{array}$ & $\begin{array}{l}13 \% \\
12 \%\end{array}$ & $\begin{array}{l}80 \mathrm{U} \\
80 \mathrm{U}\end{array}$ \\
\hline Pronation of the forearm & $\begin{array}{l}\text { Pronator teres } \\
\text { Pronator quadratus }\end{array}$ & $\begin{array}{l}11 \% \\
3 \%\end{array}$ & $\begin{array}{l}60 \mathrm{U} \\
40 \mathrm{U}\end{array}$ \\
\hline Extension of the wrist & Extensor carpi radialis & $6 \%$ & $70 \mathrm{U}$ \\
\hline Supination of the forearm & Supinator & $5 \%$ & $70 \mathrm{U}$ \\
\hline Forced flexion of the thumb and index finger & Flexor pollicis longus & $7 \%$ & $50 \mathrm{U}$ \\
\hline $\begin{array}{l}\text { Flexion of the fingers (or selective flexion of one finger, } \\
\text { for example the index finger) }\end{array}$ & $\begin{array}{l}\text { Flexor digitorum superficialis } \\
\text { Flexor digitorum profundus }\end{array}$ & $\begin{array}{l}4 \% \\
7 \%\end{array}$ & $\begin{array}{l}70 \mathrm{U} \\
70 \mathrm{U}\end{array}$ \\
\hline Extension of the thumb and index finger & $\begin{array}{l}\text { Extensor pollicis } \\
\text { Extensor indici }\end{array}$ & $\begin{array}{l}6 \% \\
5 \%\end{array}$ & $\begin{array}{l}40 U \\
40 U\end{array}$ \\
\hline $\begin{array}{l}\text { Extension of the fingers (or selective extension of } \\
\text { one finger) }\end{array}$ & $\begin{array}{l}\text { Extensor digitorum communis and } \\
\text { extensor digiti mini }\end{array}$ & $2 \%$ & $50 \mathrm{U}$ \\
\hline Shoulder elevation & $\begin{array}{l}\text { Deltoid } \\
\text { Levator scapulae } \\
\text { Supraspinatus }\end{array}$ & $\begin{array}{l}6 \% \\
4 \% \\
4 \%\end{array}$ & $\begin{array}{l}100 U \\
160 U \\
120 U\end{array}$ \\
\hline
\end{tabular}

Doses are expressed as IU Dysport (500 U/2.5 ml).

Pectoralis major, dorsal muscles, adductor pollici, and abductor pollici brevis were injected occasionally.

The effect of treatment (subjective improvement) was evaluated by the patients and reported at each routine follow up visit. This was a global clinical impression of the impact of treatment on writing quality (global effect on increase in writing speed, fewer writing errors, more legible handwriting). The best percentage of improvement obtained by the patient was defined as maximum effect of the BTX injection during the period between two injections (from the last injection to the day of the routine evaluation at the time of the next BTX injection). This improvement was rated by the patients at each visit in comparison to the disability before any BTX injection $(<20 \%, 20-50 \%, 50-80 \%,>80 \%)$.

A $20-50 \%$ improvement in quality of writing was the cut off for a clinically relevant improvement.

Objective improvement was assessed by the examiner, using the Burke-Fahn-Marsden ${ }^{13}$ (BFM) scale (disability and severity scores for the upper limb at the initial visit ("first visit") and at the time of the study ("last visit"), at maximum effect of the injection. Although the BFM scale was designed to evaluate patients with generalised dystonia, there are specific items from the upper limb in the "severity scale" and several items exploring manual function, including handwriting in the "disability scale" (see appendix). Moreover, the BFM scale has been found sensitive enough to assess focal dystonia in other studies related to upper limb dystonia. ${ }^{14}$

\section{Statistics}

BFM scores at the "first visit" and "last visit" were compared using a paired Wilcoxon sign rank test. To look for predictive factors for the efficacy of BTX injections, we used mixed models (analysis of variance (ANOVA) with random effect) with a random effect for patients and a fixed effect for tested factors (age, sex, duration of evolution, specific motor pattern, tremor, myoclonus, presence of mirror movements, muscles that were injected with BTX, number of injection sessions). These models allow analysis of the evolution of the condition in each patient. Probability $(p)$ values of less than 0.05 were considered statistically significant. Statistical analyses were undertaken using the SAS 8.1 statistical package (SAS Institute, Cary, North Carolina, USA).

\section{RESULTS}

There were 44 patients with dystonic writer's cramp and three with primary writing tremor (28 women and 19 men). Mean (SD) age was 46.1 (14.0) years (range 19 to 77 ), and the mean disease duration was 7.6 (8.1) years (range 1 to 38 ).

Abnormal postures observed during writing (alone or in combination) in the 47 patients treated with BTX were: flexion-pronation of the forearm $(51 \%)$, flexion of the fingers $(51 \%)$, flexion of the wrist $(30 \%)$, extension of the thumb and index finger $(28 \%)$, extension of the fingers and/or wrist ( $11 \%)$, shoulder elevation (23\%), supination of the forearm (17\%). Mirror movements in the dystonic hand while writing with the non-dominant hand were observed in 19\% of the patients. Some patients had dystonic tremor (13\%) or myoclonus (6\%) superimposed on the dystonic posture.

Results of BTX treatment and predictive factors for improvement in motor disability were analysed for the 47 patients treated with BTX. These patients had a combined total of 155 injection sessions (mean (SD) number of sessions per patient: 2.8 (1.9), range 1 to 11 ; duration between first and the last injection: 19 (17) months, range 6 to 84). In all, 324 muscles were injected over all the sessions.

Subjective benefit was observed by $73 \%$ of the injected patients (mean subjective improvement, 43 (3)\%). On the BFM scale, there was an improvement $(\mathrm{p}<0.0001)$ in both severity and disability scores (table 2 ) when pretreatment ("first visit") scores were compared with post-treatment scores ("last visit"), at the time of the study. There was a significant correlation between effect of treatment subjectively evaluated by the patient and objectively measured by

Table 2 Severity and disability scores of the Burke-FahnMarsden scale before and after treatment

\begin{tabular}{llll}
\hline Scores & Before treatment & After treatment & \\
\hline Severity & $2.8(1.8)$ & $1.8(1.7)$ & $\mathrm{p}<0.0001$ \\
Disability & $1.9(0.8)$ & $1.1(1.0)$ & $\mathrm{p}<0.0001$ \\
\hline
\end{tabular}

Scores are expressed as mean (SD); scores were obtained at the "first visit" and at the "last visit". 
the BFM disability score $(r=0.35, \mathrm{p}<0.04)$. A similar tendency was observed for the severity score $(r=0.31$, $\mathrm{p}>0.05$ ).

Two particular patterns of writer's cramp were good predictors of improvement in the functional disability (BFM scale) with BTX treatment: extension of the thumb $(\mathrm{p}<0.04)$ and flexion and pronation of the forearm $(\mathrm{p}<0.03)$ were associated with the most significant improvement. The effects of the two patterns were independent (table 3).

Age was a predictive factor for improvement in the severity score (BFM scale)-improvement was greater for older patients $(\mathrm{p}<0.006)$. Patients without dystonic tremor were more improved than those with dystonic tremor (BFM scores for severity $(p<0.05)$ and disability $(p<0.0008)$ ). In contrast, the presence of dystonic tremor was predictive of a poor response. Duration of disease evolution and sex did not influence the response (table 3 ).

The pattern of writer's cramp with pronation and flexion of the forearm could be associated with a flexed posture of the fingers. Thus the most active muscles were the pronator teres, the flexor carpi radialis or ulnaris, and the flexor digitorum. In keeping with the finding that this particular pattern was a good predictive factor for functional improvement, we found a significant association with the following injected muscles, and a greater subjective improvement: flexor carpis radialis $(\mathrm{p}<0.006)$, flexor digitorum profundus $(\mathrm{p}<0.003)$, association of flexor digitorum profundus and flexor digitorum communis $(\mathrm{p}<0.001)$, and shoulder muscles, mainly supraspinatus $(p<0.02)$. These effects were independent. This improvement was present from the time of the first injection and was maintained throughout the period of the injection sessions. There was a correlation between the number of sessions and the mean percentage of subjective improvement $(r=0.34, \mathrm{p}=0.03)$.

Fifty three per cent of the patients returned for at least three injection sessions, while $47 \%$ had only one or two injections, as they were more recently involved in the study. The two groups did not differ in the improvement of their disability scores (in keeping with the presence of benefit since the time of the first injection). The improvement in severity score was slightly greater in those who returned repeatedly $(\mathrm{p}<0.04)$.

\section{DISCUSSION}

In patients with writer's cramp treated with BTX injections, our study specifically addressed, for the first time, the issues of the predictive role of the pattern of dystonia and the choice of injected muscles in the treatment response. It appears from our results that patients with a pronation-flexion pattern of dystonia show the best and the most sustained improvement. This determines the selection of the muscles treated with BTX: selective EMG guided injections into the wrist and finger flexor muscles are effective at restoring motor function with very few adverse effects.

Evaluation of motor function was clinically meaningful as both patients and examiners provided ratings of the efficacy of the BTX injections. A significant $(p<0.0001)$ objective improvement was obtained for both disability and severity scores for the upper limb on the BFM scale, ${ }^{13}$ rated by the examiner. Subjective evaluation, rated by the patient, consisted of a global improvement in writing speed, legibility, and number of writing errors. In order to minimise placebo effects, ${ }^{468}$ a $20-50 \%$ improvement was the cut off for assuming a clinically relevant improvement in the patient's handwriting. A considerable (47\%) functional improvement in writing was reported by the patients. This is consistent with an increase in writing speed found in previous studies. ${ }^{8}{ }^{10}$ In keeping with results of double blind studies, ${ }^{4} 68$ concordance of subjective response and objective testing (correlation between patient's opinion and BFM scale) was observed in our study.

The number of injection sessions did not have a large influence on the final benefit, as patients experienced benefit from the time of the first injection. We found that $73 \%$ of patients with writer's cramp receiving BTX treatment experienced some subjective benefit. In earlier studies, such good results were related to short periods of follow up. ${ }^{35} 910$ However, in our series, the improvement in disability scores in the $47 \%$ of patients who had only one or two injections sessions did not differ significantly from the improvement in the $53 \%$ of patients who returned repeatedly for at least three injections sessions. In the latter group, benefit was maintained over time and the improvement in the severity score was slightly greater. This is in keeping with a prolonged and substantial benefit observed in $56 \%$ of patients with writer's cramp reported in previous series, with longer follow up periods. $^{711}$

The presence of dystonic tremor was the only factor predictive of a poor response in our study. This has been identified previously as indicative of poor response to treatment, ${ }^{16}$ among other elements of poor motor control ${ }^{15}$ such as co-contraction of antagonist muscles, tremor, and lack of selectivity in attempts to perform independent finger movements.

\section{Conclusions}

Our results should help clinicians to identify patients with writer's cramp who are likely to benefit most from BTX treatment, and to select the most appropriate injection strategy in a subset of population with pure writer's cramp.

Table 3 Predictive factors for improvement in disability and severity

\begin{tabular}{|c|c|c|c|c|c|c|}
\hline & \multicolumn{3}{|c|}{ Disability } & \multicolumn{3}{|c|}{ Severity } \\
\hline & $\bar{\beta}$ & SE & $\mathrm{p}$ Value & $\beta$ & SE & $p$ Value \\
\hline Sex & & & NS & & & NS \\
\hline Age & & & NS & 0.03 & 0.01 & $<0.006$ \\
\hline Duration of evolution & & & NS & & & NS \\
\hline Extension of the thumb & 0.56 & 0.25 & $<0.04$ & & & NS \\
\hline Flexion and pronation of forearm & 0.66 & 0.29 & $<0.03$ & & & NS \\
\hline Other affected muscles & & & NS & & & NS \\
\hline Myoclonus & & & NS & & & NS \\
\hline Mirror movements & & & NS & & & NS \\
\hline Physiotherapy & & & NS & & & NS \\
\hline Number of muscle injected (less than & & & & & & \\
\hline$v$ more than 3) & & & NS & & & NS \\
\hline
\end{tabular}

$\beta$ : parameter estimate. For categorical variables, the improvement is increased of a $\beta$ factor when the tested factor is present. For continuous variables, the improvement is increased of a $\beta$ factor per unit increase of the tested factor. 
We cannot exclude the possibility that the better outcome was linked to improved identification of the abnormal pattern and better selection of the muscles treated with BTX. Beneficial effects could reflect an improvement in abnormal posture, allowing a more normal pattern of movement, or be related to adaptive cortical reorganisation. ${ }^{17}$

\section{ACKNOWLEDGEMENTS}

We are indebted to Dr Alexis Elbaz for his helpful comments, and to Nick Barton and Veronique Leteur for editorial assistance.

\section{Authors' affiliations}

S Sangla, J S Vidal, G Gallovedec, Department of Neurology, Hôpital Saint-Antoine, Paris, France

M Vidailhet, INSERM U289, Pitié-Salpêtrière Hospital, Paris

S T du Montcel, Biostatistics Department, Pitié-Salpêtrière Hospital, Paris R Diebbari, Department of Neurosurgery, Beaujon Hospital, Paris

Competing interests: none declared

\section{APPENDIX}

\section{Burke-Fahn-Mardsen scale}

Items and subscores used to evaluate the patients with writer's cramp.

\section{Dystonia movement scale, subscore for the arm} region

Provoking factor $(0-4) \times$ severity factor $(0-4) \times$ weight $(1)$

$=$ score $(0-16)$

Provoking factors:

0, No dystonia at rest or with action

1, Dystonia on particular action

2, Dystonia on many actions

3. Dystonia on action of distant part of body or intermittently at rest

4, Dystonia present at rest

Severity factors (arm):

0 , No dystonia present

1, Slight dystonia. Clinically insignificant.

2, Mild. Obvious dystonia, but not disabling.
3, Moderate. Able to grasp, with some manual function.

4, Severe. No useful grasp.

Dystonia disability scale: subscore for handwriting (tremor or dystonia)

0 , Normal

1, Slight difficulties, legible.

2, Almost illegible.

3, Illegible.

4, Unable to grasp to maintain hold on pen.

\section{REFERENCES}

1 Sheehy MP, Rothwell JC, Marsden CD. Writer's cramp. In: Fahn S, Calne F, Marsden CD, eds. Dystonia 2. Advances in Neurology, vol 50. New York: Raven Press, 1988:457-72.

2 Cohen LG, Hallett M, Geller BD, et al. Treatment of focal dystonias of the hand with botulinum toxin injections. J Neurol Neurosurg Psychiatry 1989;52:355-63.

3 Rivest J, Lees AJ, Marsden CD. Writer's cramp: treatment with botulinum toxin injections. Mov Disord 1991;6:55-9.

4 Yoshimura DM, Aminoff MJ, Olney RK. Botulinum toxin therapy for limb dystonias. Neurology 1992;42:627-30.

5 Jankovic J, Schwartz KS. Use of botulinum toxin in the treatment of hand dystonia. J Hand Surg 1993;18:883-7.

6 Tsui JK, Bhatt M, Calne S, et al. Botulinum toxin in the treatment of writer's cramp: a double-blind study. Neurology 1993;43:183-5.

7 Karp BI, Cole RA, Cohen LG, et al. Long-term botulinum toxin treatment of focal hand dystonia. Neurology 1994:44:70-6.

8 Cole R, Hallett M, Cohen LG. Double-blind trial of botulinum toxin for treatment of focal hand dystonia. Mov Disord 1995; 10:466-71.

9 Turjanski N, Pirtosek Z, Quirk J, et al. Botulinum toxin in the treatment of writer's cramp. Clin Neuropharmacol 1996;19:314-20.

10 Wissel J, Kabus C, Wenzel R, et al. Botulinum toxin in writer's cramp: objective response evaluation in 31 patients. I Neurol Neurosurg Psychiatry 1996;61:172-5.

11 Hsiung GY, Das SK, Ranawaya R, et al. Long-term efficacy of botulinum toxin $\mathrm{A}$ in treatment of various movement disorders over a 10-year period. Mov Disord 2002; 17:1288-93.

12 Molloy FM, Shill HA, Kaelin-Lang A, et al. Accuracy of muscle localization without EMG: implications for treatment of limb dystonia. Neurology 2002;58:805-7.

13 Burke RE, Fahn S, Marsden CD, et al. Validity and reliability of a rating scale for the primary torsion dystonias. Neurology 1985;35:73-7.

14 Meunier S, Lehericy S, Garnero L, et al. Dystonia: lessons from brain mapping. Neuroscientist 2003;9:76-81.

15 Cohen LG, Hallett M. Hand cramps: clinical features and electromyographic patterns in a focal dystonia. Neurology 1988;38:1005-12.

16 Brin MF, Lyons KE, Doucette J, et al. A randomized, double masked, controlled trial of botulinum toxin type $A$ in essential hand tremor. Neurology 2001;56:1523-8.

17 Byrnes ML, Thickbroom GW, Wilson SA, et al. The corticomotor representation of upper limb muscles in writer's cramp and changes following botulinum toxin injection. Brain 1998;121:977-88. 